\title{
Is evidence for late selection due to automatic or attentional processing of stimulus identities?
}

\author{
LISA R. FOURNIER and STEPHANIE SHORTER \\ Washington State University, Pullman, Washington
}

\begin{abstract}
This study determined whether evidence for late selection is due to attention processing or to processing by an automatic system that is separate from attention (two systems framework; Eriksen, Webb, \& Fournier, 1990). The task was a two-choice discrimination of a target that appeared in one of two sequentially cued locations in an eight-lettervisual display. Attention was directed to the first cued location (cue 1), and whether identification processing occurred at a different location before the second cue (cue 2) directed attention there was determined. Cue validity varied across two experiments, and critical trials were those in which the target appeared at cue 2. For these trials, the target was preceded by a letter (either identical, neutral, or incompatible) that changed to the target at various time intervals following cue 2. Automatic identification was assumed if the incompatible letter interfered with response to the target when it appeared only before cue 2 onset and independent of cue validity. The incompatible letter appearing only before cue 2 onset interfered with the target when the target occurred equally often at cue 1 and cue 2 , but not when the target occurred at cue $170 \%$ and at cue 2 $30 \%$ of the time. This disconfirms the two systems framework and suggests that attention is required for spatial form processing and response competition.
\end{abstract}

The question of whether attention selects information for identity processing (early selection) or whether attention selects for response activation among information that is already identified (late selection) has been debated since the 1960s. Despite the considerable amount of research investigating this issue, substantial disagreement remains. A number of studies provide evidence that individual items within a multi-element, visual display are serially attended and identified. This is consistent with early selection because attention seems to be the limiting factor for spatial form processing (e.g., Averbach \& Coriell, 1961; Eriksen \& Rohrbaugh, 1970; Pashler, 1984; Sperling, 1960; Treisman \& Gelade, 1980; Yantis \& Johnston, 1990). On the other hand, many studies indicate that several items in a visual display can be identified in parallel. This is consistent with late selection because spatial form processing does not seem to be limited by attention, therefore it is assumed that attention acts later in the processing stream to select responses (e.g., Allport, 1987; Eriksen \& Spencer, 1969; Kinchla, 1974; LaBerge, 1975; Logan, 1988; Miller, 1991; Shiffrin \& Gardner, 1972; Shiffrin \& Schneider, 1977; Tipper, 1985; Tipper \& Cranston, 1985). Two frameworks have been proposed to account for the conflicting evidence regarding early versus late selection.

We thank Robert Patterson for his comments on earlier drafts of this paper, Brian P. Dyre for programming related to these experiments, and Richard Shiffrin for discussions related to this study. Correspondence concerning this article should be addressed to L. R. Fournier, Department of Psychology, Washington State University, Pullman, WA 99164-4820 (e-mail: fournier@wsunix.wsu.edu).
The first framework is the perceptual load framework proposed by Lavie and Tsal (1994). Importantly, this framework assumes that one system (i.e., attention) is responsible for identity processing and that only attended stimuli will be processed by this system. Similar to zoom lens models of attention (e.g., Eriksen \& St. James, 1986; Eriksen \& Yeh, 1985), the perceptual load framework assumes that attention is limited in capacity, with the spatial allocation of attention inversely proportional to the perceptual demands of the task. This framework further assumes that one cannot allocate less than the total amount of capacity available at one time. Thus, a task with low perceptual load will cause attention resources to "spill over" to other task-irrelevant locations, and these taskirrelevant locations will be involuntarily processed by attention. In contrast, a task with high perceptual load may require the total amount of attentional capacity available and prevent involuntary allocation of attention to taskirrelevant locations. Note, however, that this framework does not assume that the spatial allocation of attention is immune to task strategies (or even task contingencies), but that such strategies cannot always prevent involuntary processing of items at task irrelevant locations.

This framework posits that evidence supporting early selection is based on tasks that impose high attention demands (high perceptual load) and require focal attention that leads to selection of an item at the attended location only, whereas evidence for late selection is based on tasks that impose less demands on attention (low perceptual load) and allow attention to be distributed voluntarily or involuntarily across several locations, which leads to parallel selection of items at these locations (see also Kah- 
neman \& Treisman, 1984). Target identification tasks that have high perceptual load include those that display several different items that are difficult to discriminate perceptually and those that demand a relatively large amount of information about a stimulus to be processed before the required response can be executed (e.g., target compatibility cuing tasks, Eriksen \& Hoffman, 1972, 1973; Yantis \& Johnston, 1990; and pre- and post-cuing tasks, Pashler, 1984; see also Duncan, 1980; Kantowitz, 1985; Lavie, 1995; Miller, 1991; Navon, 1989; Treisman $\&$ Gelade, 1980). Target identification tasks that have low perceptual load are those that display only a few items that are easy to discriminate perceptually and require only a small amount of information about a stimulus to be processed before the required response can be executed (e.g., flankertasks, B. A. Eriksen \& C. W. Eriksen, 1974; negative priming tasks, Tipper, 1985; samedifferent matching tasks, Keren, O'Hara, \& Skelton, 1977). Results supporting the perceptual load framework come from research by Lavie (1995) and Rees, Frith, and Lavie (1997), which show that increasing perceptual load attenuates processing of task-irrelevant stimuli. However, these studies (and others that have obtained similar findings) are consistent with those demonstrating that an attended stimulus receives spatiotemporal priority and do not directly address whether unattended stimuli can be identified (see Enns \& Di Lollo, 1997; Stelmach \& Herdman, 1991; Stelmach, Herdman, \& McNeil, 1994).

The second framework is the two systems framework proposed by Eriksen, Webb, and Fournier (1990). In contrast to the perceptual load framework, this framework assumes that two systems are responsible for identity processing. Attended stimuli are assumed to be processed by an attentional system, whereas unattended stimuli are processed by an automatic system. Thus, attention is not required for identification processing. The attention system is assumed to be involved in learning, complex mental operations, and the conscious noting of stimuli and responses, whereas the automatic system is assumed to be involved in innate behaviors as well as stimulusresponse correlations that are highly learned (see criteria set forth by LaBerge, 1973, and Shiffrin \& Schneider, 1977; and see Shiffrin, 1988, for a review). Moreover, the automatic system is assumed to process stimuli in parallel even though the output of its processing (i.e., identification and response activation) may be overridden by attention. The implication of this idea is that when the attention system is directed to a stimulus, identity processing of this stimulus is largely independent of the identity processing that is occurring, or that has already occurred, in the automatic system. However, response activation that results from attention processing may be affected by response activation that results from automatic processing.

This framework posits that evidence supporting early selection is based on tasks that impose high attention demands and require focal attention, which leads to selection of an item at the attended location only. However, unlike the perceptual load framework, this framework posits that evidence for late selection is based on tasks that promote highly learned stimulus-response correlations, allowing automatic identification and response activation that is independent of attention processing. For example, visual search tasks in which targets and distractors are consistently mapped (and are perceptually dissimilar) show that target detection time is often unaffected by the number of distracters (e.g., Duncan \& Humphreys, 1989; Shiffrin \& Schneider, 1977). Also, response compatibility tasks show that if stimuli at task-irrelevant locations have highly learned stimulus-response correlations that conflict with the target response, the target response is usually delayed (e.g., Colegate, Hoffman, \& Eriksen, 1973; Eriksen \& Hoffman, 1973; Fournier, Bowd, \& Herbert, 2000). Results supporting the two systems framework come from research by Eriksen et al. (1990). They showed that response to a target could be disrupted if the original letter occupying the target location could activate a highly learned, competing response and change to the target letter before focal attention apparently shifted to this location. However, it is possible that the resulting target interference was due to attending to the original letter, not automatically identifying the original letter.

The important difference between the perceptual load and the two systems frameworks is that the former assumes attention is necessary for identity processing. Note that this assumption is not unique to the perceptual load framework, but is the main assumption of early selection models in general (e.g., spotlight and zoom lens models). The perceptual load framework is unique in that it assumes attention can be voluntarily as well as involuntarily allocated to different spatial locations, whereas most early selection models emphasize the voluntary or strategic factors governing the spatial distribution of attention (e.g., Eriksen \& St. James, 1986; Jonides, 1980, 1983; Kahneman \& Treisman, 1984; LaBerge, 1973). The perceptual load account of the early versus late selection debate, similar to the two systems account, is especially attractive because it explains why task-irrelevant stimuli are sometimes processed even though such processing can lead to poor task performance and, therefore, may have no strategic advantage. However, the distinction as to whether attention is involuntarily or voluntarily allocated to task-irrelevant locations is beyond the scope of the present study; we address the issue of whether or not identity processing requires attention.

The purpose of the present study was to evaluate the two systems account of the early versus late selection debate by determining whether an unattended stimulus can be automatically identified. To accomplish this, the methodology developed by Eriksen et al. (1990) was used because it provides a measure of the time course of stimulus identification and can potentially determine whether identification of an object occurs before focal attention is allocated to an object (Shiffrin, Diller, \& Cohen, 1995). We describe Eriksen et al.'s (1990) methods, findings, and conclusions in some detail, since we will be evaluating their conclusions by utilizing their methods. 
The goal of their study was to direct focal attention to a single stimulus location using a location cue and, while attention was engaged at this location, determine whether any identity processing automatically occurred at a different stimulus location, before attention was directed to this location by a second location cue. Two different locations were serially cued within an eight-letter, circular array. See the trial sequence and duration of events in Figure 1. The first cue (cue 1) appeared $50 \mathrm{msec}$ before letter-display onset and the second cue (cue 2) appeared $50 \mathrm{msec}$ after letter display onset. It was assumed that these cues directed focal attention serially to each cued location. Observers made a two-choice discrimination between two target letters (e.g., A and K), one of which appeared in either the first or second cued location $(50 \%$ probability at each cued location).

The trials of main interest were those in which the target appeared at the second cued location (cue 2). For these trials, the target was preceded by a letter that later changed to the target following cue 2 on set. The original letter that changed to a target was either identical, neutral, or incompatible to the target. Also, the original letter changed to a target following cue 2 onset at predetermined delays (change delays) ranging from 0 to $83 \mathrm{msec}$.

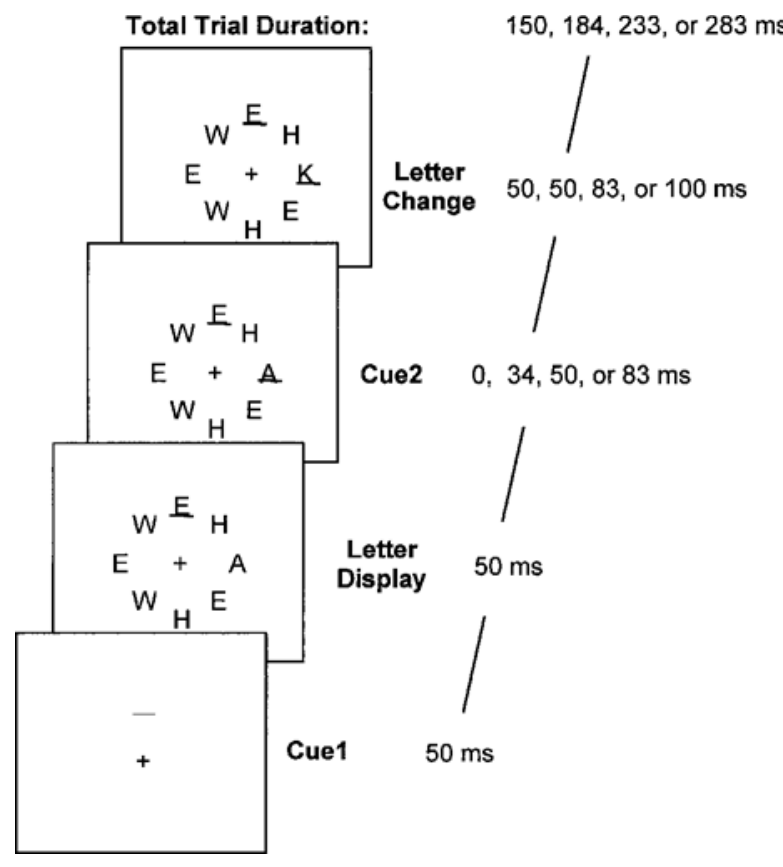

Figure 1. The sequence and duration of each display frame within a trial for the Eriksen et al. (1990) study. The letter change for the incompatible condition is shown, although the sequence and duration of display frames for the other letter change conditions were identical. The durations listed beside the cue 2 frame represent the four different delays (change delays) in which the original letter changed its identity to a target following cue 2 onset; the durations listed beside the letter change frame represent the duration of the target for the four different change delays, respectively.
Eriksen et al. (1990) posited that if the original letter was incompatible with the target and was identified before attention shifted from the cue 1 to the cue 2 location, the incompatible letter should interfere with target identification even at short change delays. This is because automatic identification of the incompatible letter should activate its associated response and compete with the alternative, target response (B. A. Eriksen \& C. W. Eriksen, 1974). Thus, when attention is directed to the cue 2 location, responses to the attended target should be delayed (and less accurate) because of interference from the original, competing response automatically activated by the incompatible letter, which must be overridden in order to respond correctly to the attended target (e.g., Coles, Gratton, Bashore, Eriksen, \& Donchin, 1985).

Eriksen et al. (1990) found that interference from the incompatible letter did not occur at short change delays (following cue 2 onset) of $0 \mathrm{msec}$ and $34 \mathrm{msec}$, suggesting that the incompatible letter was not yet identified at these short change delays, consistent with early selection models. However, interference from the incompatible letter did occur at the 50-msec change delay, whereas interference from the neutral letter occurred at the $83-\mathrm{msec}$ change delay. Because interference from the incompatible letter occurred earlier than that from the neutral letter, it was assumed that interference from these letters were not due to the same processing system. They reasoned that, if both letters had been processed by the same system (i.e., attention), the time at which identification processing would have to restart once these letters changed to the target should have been the same and therefore interference should have occurred at the same change delay. They proposed instead that early interference from the incompatible letter was due to automatic processing by a system separate from attention, whereas later interference from the neutral letter was due to attention. This proposal follows the assumption that automatic processing of a stimulus will interfere with the attended target only if the stimulus activates a competing response. Because automatic processing of the neutral letter should not activate a competing response, any interference found in the neutral condition is attributed to attention (i.e., identity processing of the neutral letter carried out by attention would have to restart once the target appeared, and time spent attending to the neutral letter would be added to that spent attending to the target). Thus, interference in the neutral condition marked the time at which attention arrived at the letter location. Moreover, because earlier interference was found for the incompatible letter, as compared with the neutral letter, response activation by the incompatible letter was attributed to automatic processing that occurred before attention was directed to the incompatible letter (i.e., automatic response activation of the incompatible letter would interfere with the response activation of the attended target). Thus, Eriksen et al. (1990) concluded that identity processing that has occurred or is occurring automatically will not necessarily disrupt responses to an attended target unless auto- 
matic identity processing leads to incompatible response activation.

There are problems, however, with the interpretation that the earlier interference from the incompatible letter was due to automatic processing. Interference from the incompatible letter occurred when it was presented for a total of $100 \mathrm{msec}(50 \mathrm{msec}$ before and $50 \mathrm{msec}$ after cue 2 onset), which was long enough for focal attention to shift from the cue 1 to the cue 2 location (at least on a proportion of trials) before the incompatibleletter changed to the new target (latency for attentional shifts has been estimated to be 50-70 msec; Eriksen, Goettl, St. James, \& Fournier, 1989; Eriksen \& Yeh, 1985; Treisman \& Gelade, 1980). If attention shifted quickly to the cue 2 location on a small proportion of these trials, this could have led to higher average RTs for the incompatible condition, relative to the neutral condition, owing to the greater magnitude of interference based on response competition.

In the present study, we investigated whether the earlier interference by the incompatible letter, relative to the neutral letter, found in the Eriksen et al. (1990) study was due to attention (e.g., perceptual load framework) or to an automatic process separate from attention (two systems framework). Stronger evidence for the two systems framework in general, and automatic processing in particular, would be obtained under the following two conditions. First, if interference by the incompatible letter occurred even when the incompatible letter was presented only before cue 2 onset (i.e., at the 0 -msec change delay), we could discount the possibility that early interference by the incompatible letter was due to cue 2 directing attention to the incompatible letter. Although Eriksen et al. (1990) did not find any interference at the 0 -msec change delay, it is possible that presenting the incompatible letter for a total duration of only $50 \mathrm{msec}$ was not sufficient time for identity processing to occur, especially because they used low contrast displays (e.g., Fournier, 1994; Hoffman, 1975; Rohrbaugh \& Eriksen, 1975). To guard against this possibility, we presented the original letters at slightly longer durations before cue 2 onset and used higher contrast displays. Second, if interference by the incompatible letter occurred independently of factors that influence the spatial allocation of attention (e.g., cue validity), we could discount the possibility that early interference by the incompatible letter was due to attention. Past research indicates that when target occurrence at a single cued location is high (high cue validity), a focal attention strategy is likely utilized, otherwise a more spatially distributed attention strategy is sometimes employed (Eriksen \& St. James, 1986; Jonides, 1980, 1983). Furthermore, Eriksen and Yeh (1985) showed that increasing target occurrence at the cue 1 location (high cue 1 validity) as opposed to the cue 2 location in a serial cuing paradigm increased the probability of serially selecting items at the cued locations in the order of cue occurrence. On the basis of these findings, we varied the spatial allocation of attention and hence the probability of serial selection, by manipulating the probability of target occurrence at the cue 1 and cue 2 locations (cue validity).

Two experiments were performed using the methods of Eriksen et al. (1990). In Experiment 1, a target appeared $50 \%$ of the time at the cue 1 location and $50 \%$ of the time at the cue 2 location, as was the case in the Eriksen et al. (1990) study. In Experiment 2, a target appeared 70\% of the time at the cue 1 location and $30 \%$ of the time at the cue 2 location. Recall that high cue 1 validity should increase the probability that the cued locations are serially attended. According to the assumptions of the two systems framework, factors that affect the spatial allocation of attention such as cue validity should not affect automatic processing. Thus, our varying cue validity should not influence the time (i.e., change delay) at which the incompatible letter degrades performance if the latter is due to automatic processing. Automatic processing of the incompatible letter should begin as soon as the incompatible letter appears, before this letter location is cued. This framework predicts that the incompatible letter preceding the target at the cue 2 location should interfere with the target at the 0 -msec change delay in both Experiment 1 and Experiment 2. In contrast, the perceptual load framework assumes that interference by the incompatible letter results from attending to this letter. Thus, varying cue validity (which has been shown to affect the spatial allocation of attention) should influence the time at which the incompatible letter degrades performance if the latter is due to attention. High cue 1 validity in Experiment 2 should increase the probability that the cued locations are serially attended and hence should reduce the probability (relative to Experiment 1) that attention is allocated to the incompatible letter location before it is cued. This framework predicts that the incompatible letter preceding the target at the cue 2 location may interfere with the target at the 0 -msec change delay in Experiment 1, but not in Experiment 2. In this case, impaired performance in the incompatible condition should occur at longer change delays in Experiment 2, as compared with Experiment 1.

\section{GENERAL METHOD}

\section{Participants}

Eighteen participants ( 3 males and 15 females) served in Experiment 1 and 17 different participants ( 8 males and 9 females) served in Experiment 2. The participants were undergraduates from Washington State University, who volunteered and received course extra credit. All were right-handed and all had normal or corrected-tonormal visual acuity as assessed by using a Snellen eye chart. In Experiment 1, 1 female participant was excused due to high error rates (e.g., 43\%) and data were analyzed for the 17 remaining participants. In Experiment 2, 3 participants ( 2 males and 1 female) were excused due to high error rates (e.g., range 39\%-58\% in some conditions) and data were analyzed for the 14 remaining participants.

\section{Apparatus and Stimuli}

Visual displays were presented on a computer monitor and were viewed binocularly through a face mask. A white fixation cross $\left(0.2^{\circ}\right)$ was presented in the center of the monitor before and during each trial. Eight letters appeared in a circular array $\left(4.82^{\circ} \times 4.66^{\circ}\right)$ around 
the fixation cross. Letters (A, K, E, W, and H) were in uppercase, colored white (mean luminance of $48.95 \mathrm{~cd} / \mathrm{m}^{2}$ ), and subtended $0.40^{\circ} \times 0.46^{\circ}$ against a dark gray background (luminance of 0.27 $\left.\mathrm{cd} / \mathrm{m}^{2}\right)$. Center-to-center distance between adjacent letters was approximately $1.63^{\circ}$.

Two letter positions were sequentially cued on each trial. Each cue consisted of two white dashes (each $0.23^{\circ}$ wide) that flanked a single letter (distance between letter and dashes were $0.35^{\circ}$ ). The two cues were separated by either two $\left(3.07^{\circ}\right)$ or three $\left(3.83^{\circ}\right)$ letter locations (cue distance variable). These two cues indicated potential target letter locations. The target letter A or K appeared in one of the two sequentially presented cue locations. The neutral letters $\mathrm{E}, \mathrm{W}$, and $\mathrm{H}$ appeared in the six noncued locations and could appear in one of the cued locations. The target and neutral letters contained similar features in order to promote focal attending of the cued letters (Eriksen et al., 1990).

A hand button held in the left hand was used to initiate trials, and a hand lever was moved with the right hand (to the right or left) to indicate the target discriminated. The computer recorded and displayed the accuracy and response time for each trial.

\section{Procedure}

The task was to discriminate which target (A or K) appeared in one of the two cued locations within the eight-letter circular array as quickly and as accurately as possible. The participants were instructed to attend to the first cued location (cue 1) and, if a target did not appear at this cue location (i.e., if instead it contained a neutral letter), they were to shift their attention to the second cued location (cue 2) to identify the target. A target always appeared in one of the cued locations on each trial. Letter locations that were not cued were to be ignored. To indicate the target discriminated, half of the participants were to move the hand lever to the right for the target A and to the left for the target $\mathrm{K}$; the other half had the opposite targetresponse assignment.

The sequence of events within a trial was as follows (see the example display in Figure 2). When the fixation cross was in clear focus, the trial was initiated by pressing the hand button. Afterwards, the first cue appeared (cue 1) and remained on the screen until the end of the trial. The circular array of letters followed cue 1 after a 57-msec delay and remained on the screen until the end of the trial. The second cue (cue 2) followed the letter display by $57 \mathrm{msec}$ and also remained on the screen until the end of the trial. Approximately $176-186 \mathrm{msec}$ after the onset of cue 2 , the entire display was turned off. The total trial duration ranged from 290-300 msec. On a proportion of the trials, a target letter occurred in the cue 1 location and a neutral letter occurred in the cue 2 location (both of which remained present until the end of the trial). On the other proportion of the trials, a neutral letter occurred in the cue 1 location and the original letter indicated by cue 2 changed its identity to a target letter following cue 2 onset (see Figure 2). The original letter that changed to a target was either identical (cue 2 control), neutral, or incompatible to the target. Letter changes occurred either 0 , 29 , or 43-44 msec after cue 2 onset (change delay). A blank screen always occurred immediately before the letter changed and thus, whether the blank screen occurred before or after cue 2 onset, was dependent on the change delay. At the 0 -msec change delay, a blank screen was inserted before cue 2 onset for a duration of $14 \mathrm{msec}$. In contrast, at the 29- and 43-44 msec change delays, a blank screen was inserted at specific time periods after cue 2 onset (but immediately before the letter change), and the duration of the blank screen was included as part of the change delay duration. ${ }^{1}$ (Referring to Figure 2, the change delay duration was calculated by summing the duration of the cue 2 frame and the duration of the succeeding blank screen frame.) On the proportion of trials in which the original letter at the cue 2 location changed to a target, the target remained on the screen until the end of the trial. Note that the duration of the target that occurred at the cue 2 location was dependent on the change delay (i.e., target durations were longer at the shorter change delays; see Figure 2). All events, including the blank screens, were identical for trials in which the target appeared in the cue 1 or cue 2 location with the exception that letter changes occurred at the cue 2 location only if the target appeared there. The observers had $2 \mathrm{sec}$ from the onset of the letter display to respond to the target identified at the cue 1 or cue 2 location, and they received reaction time (RT) and accuracy feedback after each response.

The type of letter change (including whether the target appeared at the cue 1 or cue 2 location) and the letter change delay (relative to cue 2 onset) were manipulated. The letter change conditions for target occurrences at the cue 2 location were as follows: (1) the neutral condition, in which a neutral letter changed to a target letter (e.g., W changed to K), (2) the incompatible condition, in which a target letter (incompatible) changed to the other target letter (e.g., A changed to K), and (3) the cue 2 control condition (formerly referred to as identical), in which a target letter originally appeared and did not change its identity (e.g., K replaced by K). There was also the cue 1 control condition, in which the target letter appeared at the cue 1 location. When the target appeared at the cue 2 location, the three letter change conditions occurred at change delays of either 0,29 , or 43-44 msec following cue 2 onset. When the target appeared at the cue 1 location, no letter changes occurred, but the same sequence of events for the three change delays occurred for this condition.

The trials of main interest were those in which the target appeared at the cue 2 location. Recall that for these trials, the target was preceded by a letter that later changed to the target following cue 2 onset. The cue 2 control condition (original letter identical to target) served as the baseline for determining whether the first letter was processed in the neutral and incompatible conditions. Eriksen et al. (1990) demonstrated that performance in the cue 2 control condition was constant across the different change delays and was similar to the neutral condition at short change delays. This indicated that there was no compatible priming in the cue 2 control condition in this task and that this condition served as an appropriate control (see also Hoffman, 1975). We were specifically interested in whether responses to the target would be delayed in the incompatible condition relative to the cue 2 control condition at the short change delay (i.e., $0 \mathrm{msec}$ ).

The cue 1 control condition was utilized to encourage serial attending of the cued locations. Eriksen et al. (1990) showed that performance in the cue 1 control condition was superior to that in the cue 2 control condition. This indicated that the cued locations were serially attended on a majority of the trials in this task.

The participants were not informed that the letters in the second cued location (cue 2) would change on some trials. Feedback was based on responses corresponding to the identity of the target that appeared at one of the cued locations; because both targets appeared at the cue 2 location in the incompatible condition, correct response feedback was given for either target response in this case. However, for data analyses, accuracy of response for targets appearing at the cue 2 location was based on identity of the target that appeared second in order to determine whether the original target letter (i.e., incompatible letter) interfered with RT and accuracy of the second target letter.

A blank screen was presented before a letter changed its identity in order to prevent attentional capture to the letter change location when the letter changes occurred. Thus, all letters in the display flickered before the target appeared to prevent attentional capture to the letter change location (e.g., Yantis \& Johnston, 1990). Also, the blank screen occurred in all letter change conditions (cue 1 control, cue 2 control, neutral, and incompatible) at all change delays $(0,29,43-44 \mathrm{msec})$ to insure that this factor contributed equally to each of these conditions.

Each block contained an equal number of the letter change conditions at each of the three change delays. In the incompatible con- 
Total Trial Duration:

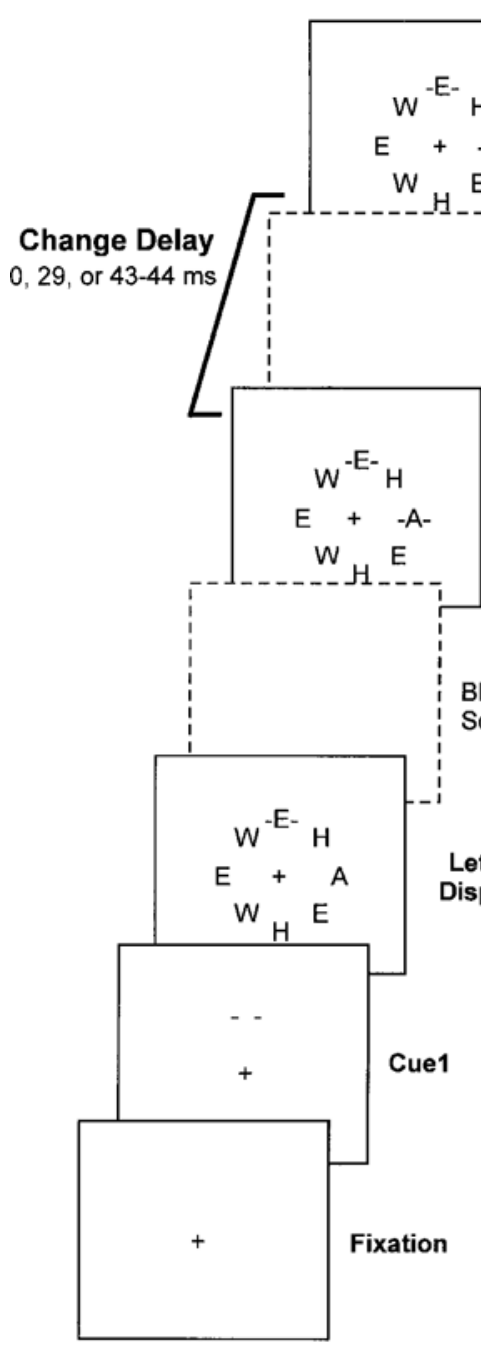

295,293 , or $290 \mathrm{~ms}$<smiles>CCCCC</smiles>

\section{Letter}

Change

167,150 , or $133 \mathrm{~ms}$<smiles>C1CCCCC1</smiles>

Blank

Screen

0,12 , or $10 \mathrm{~ms}$

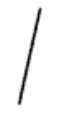

Cue2

0,17 , or $33 \mathrm{~ms}$<smiles>C1CCCC1</smiles>

14,0 , or $0 \mathrm{~ms}$

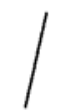

$57 \mathrm{~ms}$
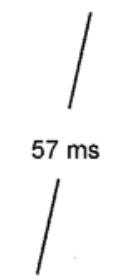

Press

handbutton

Experiment 1
299,300 , or $300 \mathrm{~ms}$<smiles>CCCC</smiles>

171,157, or $142 \mathrm{~ms}$<smiles>C1CCCCC1</smiles>

0,14 , or $14 \mathrm{~ms}$<smiles>C1CCC1</smiles>

0,15 , or $30 \mathrm{~ms}$<smiles>C1CCCCC1</smiles>

14,0 , or $0 \mathrm{~ms}$<smiles>C1CCCCC1</smiles>

$57 \mathrm{~ms}$<smiles>C1CCCCC1</smiles>

\section{$57 \mathrm{~ms}$}<smiles>C1CCCC1</smiles>

Press

handbutton

Figure 2. The sequence and duration of each display frame within a trial. The letter change for the incompatible condition is shown, although the sequence and duration of display frames for the other letter change conditions were identical. The duration of each display frame is presented separately for Experiment 1 and Experiment 2. For each experiment, the three durations listed beside each frame (succeeding the letter display frame) represent the duration of each display frame for the three different change delays of 0, 29, or 43-44 msec, respectively. The three different change delays $(0,29$, or $43-44 \mathrm{msec})$ were determined by summing the durations of the cue 2 frame and the succeeding blank screen frame. Note that only for the 0 -msec change delay did a blank screen of $14 \mathrm{msec}$ precede the onset of cue 2 .

dition, each of the two targets appeared with equal frequency as the original letter and as the target at the three change delays within a block. In the neutral condition, each possible neutral letter appeared as the original letter and was paired with each of the two targets with equal frequency at each change delay (balance was approximate across blocks, but was complete within sessions). Neutral letters that were not cued appeared randomly in the display, so that no two identical letters appeared in adjacent locations.

The letter locations that were cued were randomly determined across trials, and the frequency in which each cue appeared at each of the eight locations was approximately equal within a block. The two levels of cue distance (i.e., cues separated by two or three letter locations) occurred in a random order and were balanced within a block. The direction of serial cuing was determined randomly with clockwise and counterclockwise directions occurring equally often within each block. Because the direction of serial cuing was randomly determined and the cue distance varied, this prevented predictability of the cue 2 location. Each experimental session consisted of six blocks of 72 trials. Data from the first session were considered practice and were not included in the final analyses.

\section{EXPERIMENT 1}

The purpose of Experiment 1 was to determine at which change delay the incompatible letter interfered 
with responses to the target when the probability of a target appearing at the cue 1 or cue 2 location was equated (i.e., each cue was $50 \%$ valid). Although cue validity was only $50 \%$, serial cuing should guide attention first to the cue 1 and then to the cue 2 location on a majority of the trials (cf. Eriksen et al., 1990).

\section{Method}

Figure 2 (Experiment 1) shows a timeline of the sequence of events within a trial for the letter change conditions (cue 1 control, cue 2 control, neutral, and incompatible) at each of the letter change delays $(0,29,43 \mathrm{msec}$, respectively). After initiating the trial, cue 1 appeared and was followed $57 \mathrm{msec}$ later by the letter display. The total duration of cue 1 and the letter display was $57 \mathrm{msec}$. Then, cue 2 appeared at a different display location either immediately or following a 14-msec blank screen in the 0-msec change delay condition only. On $50 \%$ of the trials, a target letter appeared at the cue 1 location. On the other $50 \%$ of the trials, the original letter indicated by cue 2 changed its identity to a target letter either 0,29 , or $43 \mathrm{msec}$ after cue 2 onset. The change delay of $29 \mathrm{msec}$ included a $17-\mathrm{msec}$ interval in which the original letter appeared with cue 2 and a 12-msec blank screen; the change delay of $43 \mathrm{msec}$ included a 33 msec interval in which the original letter appeared with cue 2 and a 10-msec blank screen. (As shown in Figure 2, the change delay duration was calculated by summing the duration of the cue 2 frame and the duration of the succeeding blank screen frame.) Following the blank screen, the target appeared in the cue 2 location for 167, 150 , or $133 \mathrm{msec}$ in the 0 -, 29-, and 43-msec change delay conditions, respectively. Also, the total trial duration was 295, 293, and $290 \mathrm{msec}$ for the 0-, 29-, and 43-msec change delays, respectively. The participants completed four, 1-h sessions over a 1-week period.

\section{Results}

Figure 3 shows the correct target discrimination RT and errors in each of the letter change conditions (cue 1 control, cue 2 control, neutral, and incompatible) at each letter change delay $(0,29$, and $43 \mathrm{msec})$. It is evident that target performance was best when the target appeared in the first cued location (cue 1 control), as compared with the second cued location (cue 2 control). Moreover, when the target appeared in the second cued location, target performance in the incompatible condition was hindered at the 0 -msec change delay relative to the neutral and cue 2 control conditions. Four-way, repeated measures analyses of variance (ANOVAs) [session $(3) \times$ cue distance $(2) \times$ letter change $(4) \times$ change delay (3)] were conducted on mean correct RTs and errors. All significant effects are presented in Table 1 .

Cue effects. Consistent with expectations and findings by Eriksen et al. (1990), RT for the cue 1 control was faster (by approximately $35 \mathrm{msec}$ ) than for the cue 2 control condition (Tukey, $p<.05$ ), which indicates that cued items were identified serially, in the order of cue presentation, on a majority of the trials. The main effect of cue distance was due to a 3 -msec increase in RT when the two cues were close in proximity $\left(3.07^{\circ}\right.$ visual angle apart) as opposed to further apart $\left(3.83^{\circ}\right.$ visual angle apart). Perhaps the onset of cue 2 caused a slight disruption in target processing when these two cues occurred closer together. ${ }^{2}$ Importantly, increased distance between cues did not lead to longer target discrimination times
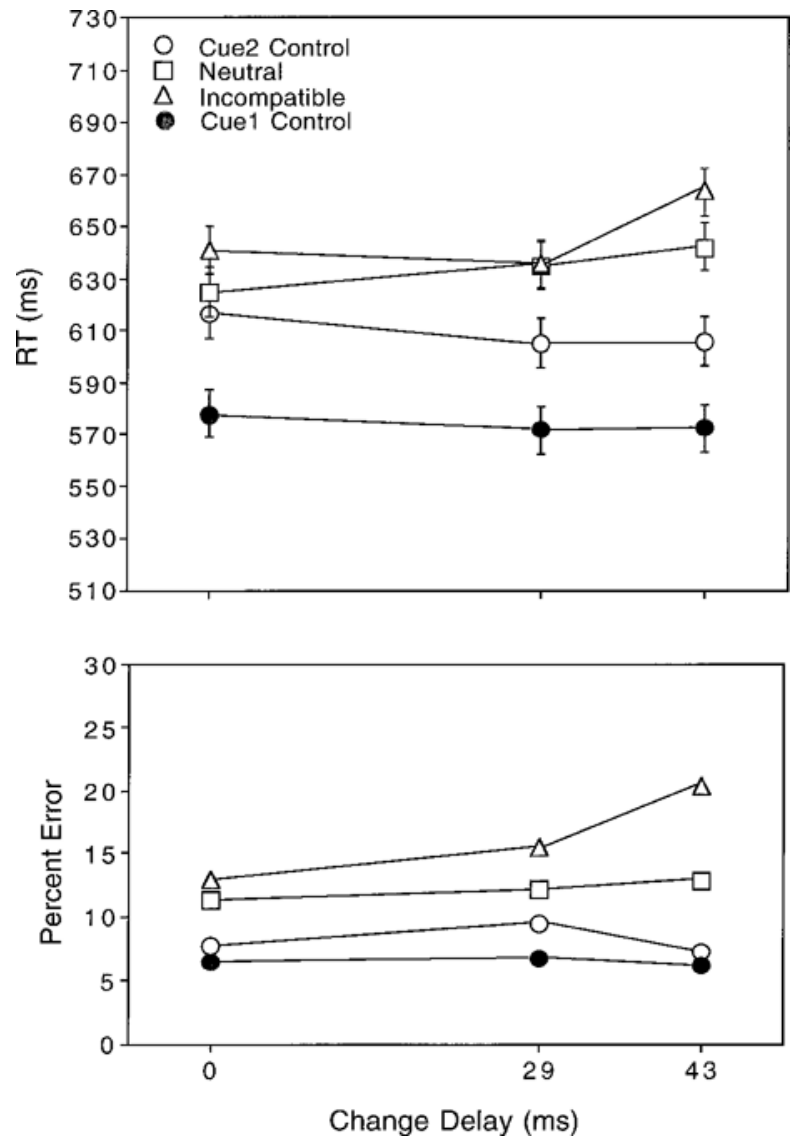

Figure 3. Correct target discrimination reaction time and error rates for each of the letter change conditions at each change delay in Experiment 1.

(similar to Eriksen et al., 1990), which indicates that attention shift times between cues did not increase at the longer cue distance (Eriksen \& Webb, 1989).

Practice effects. The session $\times$ letter change interaction for RT revealed that RT decreased for all of the letter change conditions in session 2 relative to session 1 (Tukey, $p \mathrm{~s}<.05$ ). Also, the RT in session 3 was no different than that in session 2 for the control conditions (cue 1 and cue 2 control) and the neutral condition; however, RT was greater in session 3 relative to session 2 for the incompatible condition (Tukey, $p<.05$ ). The slower RTs in session 3 for the incompatible condition might have been due to the higher accuracy found for this condition in session 3 (3\% and 5\% fewer errors) relative to sessions 1 and 2 [note the session 3 letter change interaction for errors approached significance, $F(6,96)=1.98, p=$ .075]. The latter findings suggest that, with practice, the participants were more likely to identify and withhold responding to the incompatible letter and respond on the basis of the new target. (Recall that responding to the incompatible letter was recorded as an error.) This would lead to slower responding to the new target because of response competition by the incompatible letter, but would also lead to more accurate target responses. 
Table 1

Correct Response Time and Accuracy Analysis of Variance Tables for Significant Effects in Experiment 1

\begin{tabular}{lrr}
\hline \multicolumn{1}{c}{ Factor } & \multicolumn{1}{c}{ Significance } \\
\hline & Response Time & \\
Session & $F(2,32)=3.32, p<.049$ & 26,383 \\
Distance & $F(1,16)=5.36, p<.034$ & 635 \\
Change & $F(3,48)=45.83, p<.001$ & 6,792 \\
Change delay & $F(2,32)=4.72, p<.016$ & 1,795 \\
Session $\times$ change & $F(6,96)=3.51, p<.003$ & 1,540 \\
Change $\times$ change delay & $F(6,96)=6.05, p<.001$ & 1,444 \\
& Accuracy & \\
Change & $F(3,48)=14.14, p<.001$ & .0409 \\
Change delay & $F(2,32)=4.22, p<.024$ & .0096 \\
Change $\times$ change delay & $F(6,96)=4.40, p<.001$ & .0090 \\
\hline
\end{tabular}

Letter change effects. The data of main interest concerned the effect of the letter change delay on target discrimination performance in the incompatible and neutral conditions relative to the cue 2 control condition. There was a significant interaction of letter change $\times$ change delay for both RT and accuracy. As shown in Figure 3, RT and error rates in the incompatible condition were greater than those in the cue 2 control condition across all change delays, and RT and error rates were greatest in the incompatible condition at the 43-msec change delay (Tukey, $p \mathrm{~s}<.05$ ). This indicates that, even before the incompatible letter was cued, it interfered with correct responses to the target. Also, lengthening the presentation time of the incompatible letter after the onset of cue 2 led to greater interference in responding correctly to the target. Furthermore, increases in RT for the neutral condition relative to the cue 2 control condition did not occur until a change delay of $29 \mathrm{msec}$; although RT did not increase further at the 43-msec change delay, error rate did increase at this change delay (Tukey, $p \mathrm{~s}<.05$ ). This indicates that the neutral letter did not interfere with correct responses to the target unless it continued to appear after cue 2 onset, and this interference increased the longer that the neutral letter was present.

The most important result is that the incompatible condition, and not the neutral condition, showed increased RT and error rates relative to the cue 2 control at the 0 $\mathrm{msec}$ change delay. This finding indicates that the incompatible letter was identified after being present for only $57 \mathrm{msec}$ and before this letter location was cued. The failure to find RT and error rate increases for the neutral condition relative to the cue 2 control at the 0 -msec change delay indicates that only the incompatible letter interfered with target processing before the onset of the second cue. Also, because no difference in performance was found between the neutral and cue 2 control conditions at the $0 \mathrm{msec}$ change delay, the performance decrements found for the incompatible condition at this change delay cannot be due to visual masking. The RT increase for the neutral condition at the $29 \mathrm{msec}$ change delay indicates that the neutral letter did not interfere with the target response until it was cued, which suggests that the neutral letter did not interfere with the target response unless the former was selected before the latter.

\section{EXPERIMENT 2}

Increases in target RT and error rates by the incompatible letter appearing only before cue 2 onset and earlier increases in target RT and error rates for the incompatible relative to the neutral condition are consistent with the predictions of the two systems framework. This framework assumes that the automatic system will begin identifying the incompatible letter as soon as it appears, even before attention is directed to this letter location by cue 2. Automatic identification of the incompatible letter appearing only before cue 2 onset should lead to response competition (B. A. Eriksen \& C. W. Eriksen, 1974) and hence interfere with the correct response to the attended target. It is possible, however, that the increases in target RT and error rates in the incompatible condition at the 0 -msec change delay were due to attending to the incompatible letter as opposed to automatic processing of the incompatible letter. The participants in Experiment 1 and those in Eriksen et al.'s (1990) study may not have directed focal attention serially to both cued locations on $100 \%$ of the trials when cue 1 validity was only $50 \%$. For example, the participants may have, at least on a proportion of trials, spread attention across the letter locations before the onset of cue 2 or failed to allocate attention to the cue 1 location and by chance allocated focal attention to the letter location that was later indicated by cue 2 .

If such a distributed or focal attention strategy was utilized on a proportion of trials in Experiment 1, the increase in cue 1 validity in Experiment 2 should increase the frequency at which focal attention is serially directed to the cue 1 and the cue 2 locations. This is because the directing of focal attention to the stimulus at the cue 1 location should lead to faster discrimination of this stimulus (e.g., Eriksen \& St. James, 1986) and, on a majority of the trials, should lead to faster target discrimination responses (large benefits). Only on a small proportion of trials will focal attention have to be shifted from the cue 1 to the cue 2 location in order to discriminate the target (small costs). Evidence that the increase in cue 1 validity will promote a focal attention strategy at the cue 2 location in a serial cuing task is provided by Eriksen and Yeh (1985); they sequentially cued two locations within a four-letter circular array. The cue 1 location contained the target on $70 \%$ of the trials, and the target appeared equally often in the cue 2 and two noncued locations on the remaining $30 \%$ of the trials. They found that discrimination RTs were fastest for targets appearing at the cue 1 location relative to the cue 2 location, but targets appearing at the cue 2 location were discriminated faster than targets appearing at the noncued locations. These results suggest that the cued locations were focally attended in the order of cue appearance; otherwise, similar RTs should 
have been found when the target appeared at the cue 2 and noncued locations.

\section{Method}

The purpose of Experiment 2 was to increase cue 1 validity in order to increase the probability of focal attending to the cued locations in the order of cue appearance. The target letter appeared at the cue 1 location on $70 \%$ of the trials and at the cue 2 location on $30 \%$ of the trials. If early interference by the incompatible letter found in Experiment 1 was due to attention (e.g., distributing attention among multiple item locations before cue 2 onset or, by chance, focally attending to the location later indicated by cue 2), we should no longer find greater RT and error rates for the incompatible condition relative to the cue 2 control condition (or to the neutral condition) at the 0 -msec change delay.

The letter display, letter change conditions, and change delays were similar to those in Experiment 1 (see Figure 2, Experiment 2). Importantly, the presentation duration of the original letters for the 0 -msec change delay was identical to that in Experiment 1. Also, the presentation duration of the original letters for the 29- and 44msec change delays differed between the two experiments by $3 \mathrm{msec}$ at most. $^{3}$

After initiation of the trial, cue 1 appeared and was followed $57 \mathrm{msec}$ later by the letter display. The total duration of cue 1 and the letter display was $57 \mathrm{msec}$. Then, cue 2 appeared at a different display location either immediately or following a 14-msec blank screen in the 0 -msec change delay condition only. On $70 \%$ of the trials, a target letter appeared at the cue 1 location. On the other $30 \%$ of the trials, the original letter indicated by cue 2 could change its identity to a target letter either 0,29 , or $44 \mathrm{msec}$ after cue 2 onset. The change delay of $29 \mathrm{msec}$ included a 15 -msec interval in which the original letter appeared with cue 2 and a 14-msec blank screen; the change delay of $44 \mathrm{msec}$ included a $30-\mathrm{msec}$ interval in which the original letter appeared with cue 2 and a 14-msec blank screen. (As shown in Figure 2, the change delay duration was calculated by summing the duration of the cue 2 frame and the duration of the succeeding blank screen frame.) Following the blank screen, the target appeared in the cue 2 location for 171,157 , and $142 \mathrm{msec}$ in the 0-, 29-, and 44-msec change delay conditions, respectively. Also, total trial durations were 299, 300, and $300 \mathrm{msec}$, for the 0-, 29-, and 44-msec change delays, respectively.

In order to balance the number of observations for the letter change conditions at the cue 2 location across experiments, the number of sessions was increased, as compared with Experiment 1. The observers completed seven 1-h sessions over a 2-week period.

\section{Results}

Figure 4 shows the correct target discrimination RT and errors in each of the letter change conditions at each letter-change delay. It is evident that target performance was best when the target appeared in the first cued location (cue 1 control) compared with the second cued location (cue 2 control). Moreover, when the target appeared at the second cued location, target performance in the incompatible condition was no longer hindered at the 0 -msec change delay relative to the neutral and cue 2 control conditions. Four-way repeated measures ANOVAs [session (6) $\times$ cue distance $(2) \times$ letter change $(4) \times$ change delay (3)] were conducted on mean correct RT and errors. All significant effects are presented in Table 2.

Cue effects. In the cue 1 control condition, the RT was approximately $90 \mathrm{msec}$ faster and the error rate was $9 \%$ lower, as compared with the cue 2 control condition (Tukey, $p s<.05$ ), which suggests that the cued items
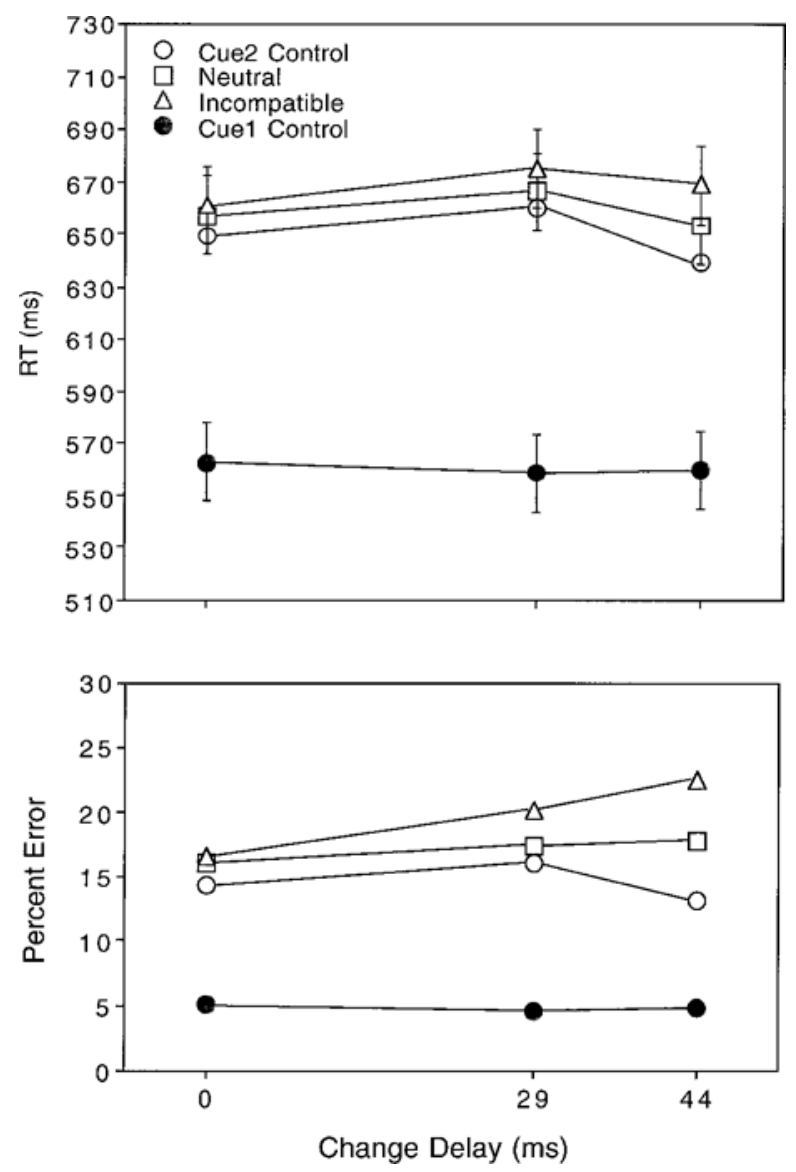

Figure 4. Correct target discrimination reaction time and error rates for each of the letter change conditions at each change delay in Experiment 2.

were identified serially, in the order of cue presentation, on a majority of the trials. In addition, the difference in RT between the cue 1 control and cue 2 control conditions was significantly greater than that found in Experiment 1 [experiment $\times$ letter change $\times$ change delay; $F(6,174)=2.2, p<.05]$; compare Figures 2 and 3 . This indicates that identification of the target was delayed longer when it appeared at the cue 2 location as opposed to the cue 1 location in this experiment relative to Experiment 1 . Taken together, these findings suggest that the cued locations were serially attended more often in the present experiment, and attending to the first cued location sometimes prevented focal attention from identifying the target at the second cued location before the display disappeared.

Practice effects. The session $\times$ change delay interaction for accuracy indicated that errors for the 44-msec change delay were slightly higher in sessions 3 and 6 . Also, the session $\times$ cue distance $\times$ letter change interaction for RT indicated that RT was greater $(7 \mathrm{msec}$ on average) when the two cues were close in proximity as opposed to further apart; however, this RT trend was re- 
Table 2

Correct Response Time and Accuracy Analysis of Variance Tables for Significant Effects in Experiment 2

\begin{tabular}{lrr}
\hline \multicolumn{1}{c}{ Factor } & \multicolumn{1}{c}{ Significance } \\
\hline & Response Time & \\
Session & $F(5,65)=12.52, p<.001$ & 24,014 \\
Distance & $F(1,13)=11.89, p<.004$ & 1,687 \\
Change & $F(3,39)=75.83, p<.001$ & 16,539 \\
Change delay & $F(2,26)=6.49, p<.005$ & 2,646 \\
Session $\times$ distance & $F(15,195)=1.72, p<.050$ & 2,547 \\
$\quad$ & & \\
& & \\
& Accuracy & \\
Distance & $F(1,13)=12.16, p<.004$ & .0113 \\
Change & $F(3,39)=37.83, p<.001$ & .0559 \\
Change delay & $F(2,26)=3.76, p<.037$ & .0134 \\
Session $\times$ change delay & $F(10,130)=1.99, p<.040$ & .0196 \\
Change $\times$ change delay & $F(6,78)=3.29, p<.006$ & .0148 \\
\hline
\end{tabular}

versed for the incompatible condition only in session 1 . We have no interpretation for these effects.

Letter change effects. The data of main interest concerned the effect of the letter-change delay on target discrimination performance in the incompatible and neutral conditions relative to the cue 2 control condition. As is shown in Figure 4, there were no RT differences among the incompatible, neutral, and cue 2 control conditions (Tukey, $p \mathrm{~s}>.05$ ) at any of the change delays (i.e., no letter change $\times$ change delay interaction). Also, accuracy did not significantly differ among the incompatible, neutral, and cue 2 control conditions until a change delay of $44 \mathrm{msec}$ (letter change $\times$ change delay interaction). At the 44-msec change delay, error rates were greater in both the incompatible and neutral conditions relative to the cue 2 control condition, and error rates in the incompatible condition were greater than those in the neutral condition (Tukey, $p \mathrm{~s}<.05$, respectively). These results indicate that the incompatible and neutral letters did not interfere with correct responding to the target at the $0 \mathrm{msec}$ or 29 -msec change delays. The incompatible and neutral letters interfered with the correct response to the target only at the 44-msec change delay, when these letters appeared $57 \mathrm{msec}$ before and $30 \mathrm{msec}$ after cue 2 onset (i.e., total presentation time of $87 \mathrm{msec}$ ). Thus, when the target had a higher probability of occurring at the first cued location, we no longer obtained interference from the incompatible letter when it was present only before the onset of the second cue.

Compatibility effects across experiments. To determine whether interference by the incompatible letter was significantly reduced in Experiment 2 (high cue 1 validity) relative to Experiment 1 (low cue 1 validity), difference scores (for RT and error rate) between the incompatible and the cue 2 control conditions were calculated at each change delay and were compared across experiments. The mixed design ANOVA [experiment $\times$ change delay (3)] conducted on the correct mean RT difference scores revealed a main effect of experiment $[F(1,29)=$ $9.51, p<.05]$ and a main effect of change delay $[F(2,58)=$
$8.39, p<.05]$. Importantly, the RT difference between the incompatible and the cue 2 control conditions was significantly greater in Experiment 1 relative to Experiment 2 , at all change delays. This confirms that the interference by the incompatible letter was significantly reduced in Experiment 2 (high cue 1 validity) relative to Experiment 1 (low cue 1 validity). Also, RT differences between the incompatible and cue 2 control conditions increased as the change delay increased, once again indicating that the incompatible letter more often interfered with the target when it remained present in the display after cue 2 onset. There were no significant effects based on error rate.

Summary. We failed to obtain evidence for automatic identity processing of letters at noncued locations when the probability was low that these noncued locations contained a target. Furthermore, the two systems framework does not predict that identity processing by the automatic system should depend on cue validity (across experiments). We found that cue validity does influence how early the incompatible letter is identified and, importantly, whether this letter is identified before its location is cued. Thus, our data disconfirm the two systems framework. That is, evidence for late selection does not appear to be based on an automatic system that is separate from attention.

\section{GENERAL DISCUSSION}

To evaluate the two systems account of the early versus late selection debate, two experiments were conducted to determine whether a visual stimulus can be identified and activate a response before attention is allocated to its spatial location. To guide the allocation of attention and examine the time course of identity processing, we used the serial cuing, response compatibility paradigm developed by Eriksen et al. (1990). Attention was directed to a single stimulus location by a location cue (cue 1) and, while attention was engaged at this location, we determined whether any identification processing occurred at a different stimulus location, before attention was directed to this location by a second location cue (cue 2). We manipulated the time at which an incompatible letter changed to a target at the cue 2 location and measured at which change delays after cue 2 onset the incompatible letter interfered with the target. We also manipulated cue 1 validity (from low to high values) because, on the basis of previous research (e.g., Eriksen \& Yeh, 1985; Jonides 1980, 1983), high cue 1 validity should increase the probability that attention is serially allocated to the cued-letter locations in the order of cue occurrence, whereas low cue 1 validity should decrease this probability.

Recall that the perceptual load framework assumes that a stimulus can only be identified if attention is allocated to its spatial location, whereas the two systems framework assumes that a task-relevant stimulus with a highly learned response can be automatically identified (without attention) when attention is engaged at a differ- 
ent stimulus location. Both frameworks assume that cue validity affects the distribution of spatial attention, but the two systems framework assumes that automatic processing is unaffected by cue validity. Thus, automatic identification of an unattended stimulus was assumed to occur if the incompatible letter preceding the target at the cue 2 location interfered with the target when the incompatible letter was present only before cue 2 onset (i.e., 0 -msec change delay) and if this early interference by the incompatible letter occurred independently of the cue 1 validity manipulations.

Indeed, in Experiment 1, in which cue 1 validity was low (i.e., 50\%), the incompatible letter interfered with the target indicated by cue 2 even when the incompatible letter was present only before cue 2 onset, providing tentative evidence for automatic processing. However, in Experiment 2, in which cue 1 validity was high (i.e., 70\%), the incompatible letter failed to interfere with the target indicated by cue 2 when the incompatible letter was present only before cue 2 onset. In this case, the incompatible letter did not interfere with the target after being present for $57 \mathrm{msec}$ prior to cue 2 onset, as was the case in Experiment 1. In fact, neither the incompatible letter nor the neutral letter interfered with the target in Experiment 2 unless these letters were present $57 \mathrm{msec}$ before cue 2 onset and remained present an additional $30 \mathrm{msec}$ after cue 2 onset. The lack of interference by the incompatible letter when it appeared only before the second cue in Experiment 2 was not likely due to perceptual factors because interference by the incompatible letter was found in Experiment 1 under identical perceptual conditions (same letter displays, letter changes, and change delay) in which only cue validity differed. It appears that cue validity influenced whether the incompatible letter was identified before its location was cued. This suggests that the incompatible letter was not identified by an automatic process separate from attention and therefore is inconsistent with the two systems framework.

Our results suggest that interference by the incompatible letter in Experiment 1 and Experiment 2 was due to attention, consistent with the perceptual load framework and early selection models in general (e.g., zoom lens and spotlight models). It appears that the lower cue 1 validity in Experiment 1 increased the probability that attention was allocated to other letter locations (i.e., including the incompatible letter location) before the second cue appeared, whereas the higher cue 1 validity in Experiment 2 decreased the probability that attention was allocated to other letter locations before the second cue appeared. Consistent with this idea, we found that the cued locations in Experiment 2 were serially identified more often, as compared with Experiment 1. Specifically, target discrimination in Experiment 2 was faster when the target appeared at the first cued location compared with the second cued location (i.e., cue 1 vs. cue 2 control conditions), and the difference in discrimination time between targets appearing at the first versus second cued locations was greater than that found in Experiment 1.
Also, in Experiment 2, the error rate was much higher for targets appearing at the second cued location relative to the first cued location (i.e., cue 2 vs. cue 1 control conditions). Taken together, it appears that high cue 1 validity $(70 \%)$ led to serial attending of the cued locations more often compared with the low cue 1 validity (50\%) and, in turn, more often led to delays in attending to and identifying the stimulus in the second cued location. Thus, decreased frequency of serial attending can account for the early interference by the incompatible letter found in our Experiment 1 (low cue 1 validity), and increased frequency of serial attending can account for the later interference by the incompatible letter in Experiment 2 (high cue 1 validity). Early interference by the incompatible letter in the Eriksen et al. (1990) study can also be accounted for by a decreased frequency in serial attending since in their experiments the same cue 1 validity was used as in our Experiment 1.

Not only do our results fail to support the two systems framework, we failed to find any evidence that a stimulus at a preattentive location receives significant form or identification processing, which is inconsistent with late selection models. According to late selection accounts, all letters begin form and identification processing in parallel as soon as the visual display is presented, independent of the allocation of attention. Thus, changing a neutral or incompatible letter to a target with the onset of the second cue should have disrupted target identification at the second cued location, independent of cue 1 validity. This is because form and identity processing that had been established at this location would have to restart when the target letter appeared with the onset of the second cue, and hence directing one's attention to a location where letter identity recently changed should delay identity processing. Our results showed that when cue 1 validity was high (Experiment 2), the uncued incompatible or neutral letter that preceded the target at the cue 2 location did not delay identification of the target (i.e., relative to the cue 2 control). Thus, when attention was directed to the cue 2 location, there was no evidence that any information at this location was processed prior to the onset of cue 2. In fact, neither the incompatible letter nor the neutral letter interfered with the target in Experiment 2 unless these letters were present $57 \mathrm{msec}$ before cue 2 onset and remained present an additional $30 \mathrm{msec}$ after cue 2 onset, which was long enough for attention to shift from the cue 1 to the cue 2 location and to identify the letter at the cue 2 location before the target appeared (e.g., Eriksen et al., 1989; Eriksen \& Yeh, 1985; Treisman \& Gelade, 1980). Thus, these findings suggest, consistent with early selection models, that identity processing does not occur until a stimulus is attended. In fact, these findings suggest that very little processing, if any, occurred before attention arrived at the letter location.

Because we failed to obtain evidence of identity processing at noncued locations (which were later cued and attended) when the probability of serially attending to the cued locations was increased, we suggest that stimuli 
with highly learned response correlations are not identified automatically by a system separate from attention (see also Rees, Russell, Frith, \& Driver, 1999). Previous studies that promoted highly learned stimulus-response correlations and showed that multiple stimuli may be identified in parallel (e.g., consistent mapping visual search tasks, Duncan \& Humphreys, 1989; Shiffrin \& Schneider, 1977; and some response compatibility tasks, Colegate et al., 1973; Eriksen \& Hoffman, 1973; Fournier et al., 2000; and flanker priming tasks, Miller, 1987; Schmidt \& Dark, 1999) likely encouraged distributed attention processing among multiple item locations (Kahneman \& Treisman, 1984; Lavie \& Tsal, 1994). It is possible that stimuli with highly learned stimulus-response correlations may reduce attention demands by decreasing the information required to identify task-relevant stimuli, which in turn may free up attention resources and allow attention to be involuntarily (or voluntarily) distributed among multiple items. Also, other factors pointed out by Lavie and Tsal, such as stimulus discriminability or the number of unique stimuli, appear to influence the resource allocation of attention. Furthermore, we show that task contingencies such as cue validity may also affect the resource allocation of attention in a task where perceptual factors are held constant (see also Eriksen \& St. James, 1986; Jonides, 1980, 1983).

We conclude that contributions of more spatially spread attention (which leads to parallel selection of items at multiple locations) may be confounded with measures of focal attention (which leads to serial selection of items) in research on the early versus late selection debate. Consistent with the perceptual load account, evidence for identity processing at task-irrelevant locations (used to support late selection) may be due to more spatially spread attending across multiple item locations, although the lack of evidence for identity processing at these locations (used to support early selection) may be due to focal attending the task-relevant location. This account is also consistent with the spotlight and zoom lens models of attention that assume spatial form and identification processing occur along a continuum (e.g., Eriksen \& Yeh, 1985) based on the distribution of attention resources among these spatial forms which, in turn, is determined by the demands of the task (e.g., Eriksen \& St. James, 1986). Thus, we suggest that parallel form and identification processing is attention limited, although the demands on attention may be quite small.

In summary, our results are inconsistent with the two systems explanation of the early selection versus late selection debate. That is, early identity processing of an incompatible letter was based on attention and not on automatic processing. Evidence supporting either early or late selection models was dependent on cue validity, which should have affected the distribution of attention but not automatic processing. It is likely that cue validity affected the mode of attention processing and hence which selection model was supported; high cue validity often led to focal attention and evidence consistent with early selection, and low cue validity sometimes led to more distributed spatial attention and evidence consistent with late selection. Thus, in accordance with the perceptual load framework and other space-based resource models, we conclude that evidence for early and late selection models is dependent on the distribution of attention. Furthermore, our findings indicate, in line with early selection models, that attention appears to be a limiting factor of identity processing and response competition.

\section{REFERENCES}

AlLPORT, A. (1987). Selection for action: Some behavioral and neurophysiological considerations of attention and action. In H. Heuer \& A. F. Sanders (Eds.), Perspectives on perception and action (pp. 395415). Hillsdale, NJ: Erlbaum.

Averbach, E., \& Coriell, A. S. (1961). Short-term memory in vision. Bell System Technical Journal, 40, 309-328.

Colegate, R. L., Hoffman, J. E., \& Eriksen, C. W. (1973). Selective encoding from multielement visual displays. Perception \& Psychophysics, 14, 217-224.

Coles, M. G. H., Gratton, G., Bashore, T. R., Eriksen, C. W., \& Donchin, E (1985). A psychophysiological investigation of the continuous flow model of human information processing. Journal of Experimental Psychology: Human Perception \& Performance, 11, 529-553.

Duncan, J. (1980). The locus of interference in the perception of simultaneous stimuli. Psychological Review, 87, 272-300.

Duncan, J., \& Humphreys, G. (1989). Visual search and stimulus similarity. Psychological Review, 96, 433-58.

EnNs, J. T., \& Di Lollo, V. (1997). Object substitution: A new form of masking in unattended visual locations. Psychological Science, 8, 135-139.

ERIKSEn, B. A., \& EriKsen, C. W. (1974). Effects of noise letters upon the identification of a target letter in a nonsearch task. Perception \& Psychophysics, 16, 143-149.

Eriksen, C. W., Goettr, B., St. James, J. D., \& Fournier, L. R. (1989). Processing redundant signals: Coactivation, divided attention, or what? Perception \& Psychophysics, 45, 356-370.

ERIKSEn, C. W., \& Hoffman, J. E. (1972). Temporal and spatial characteristics of selective encoding from visual displays. Perception \& Psychophysics, 12, 201-204.

ERIKSEn, C. W., \& Hoffman, J. E. (1973). The extent of processing of noise elements during selective encoding from visual displays. Perception \& Psychophysics, 14, 155-160.

Eriksen, C. W., \& Rohrbaugh, J. W. (1970). Visual masking in multielement displays. Journal of Experimental Psychology, 83, 147-154.

ERIKSEN, C. W., \& SPENCER, T. (1969). Rate of information processing in visual perception: Some results and methodological considerations. Journal of Experimental Psychology Monographs, 79, 1-16.

ERIKSEN, C. W., \& ST. JAMES, J. D. (1986). Visual attention within and around the field of focal attention: A zoom lens model. Perception \& Psychophysics, 40, 225-240.

ERIKSEn, C. W., \& WebB, J. M. (1989). Shifting of attentional focus within and about a visual display. Perception \& Psychophysics, 45, $175-183$.

Eriksen, C. W., Webb, J. M., \& Fournier, L. R. (1990). How much processing do nonattended stimuli receive? Apparently very little, but ... Perception \& Psychophysics, 47, 477-488.

ERIKSEN, C. W., \& YEH, Y. (1985). Allocation of attention in the visual field. Journal of Experimental Psychology: Human Perception \& Performance, 11, 583-597.

Fournier, L. R. (1994). Selective attentional delays and attentional capture among multiple visual onset elements. Perception \& Psychophysics, 56, 536-550.

Fournier, L. R, Bowd, C., \& Herbert, R. (2000). Interference from multi-dimensional objects during feature and conjunction discriminations. Quarterly Journal of Experimental Psychology, 53A, 191209. 
Hoffman, J. E. (1975). Hierarchical stages in the processing of visual information. Perception \& Psychophysics, 18, 348-354.

JONIDES, J. (1980). Towards a model of the mind's eye's movement. Canadian Journal of Psychology, 34, 103-112.

JoNIDES, J. (1983). Further toward a model of the mind's eye's movement. Bulletin of the Psychonomic Society, 21, 247-250.

Kahneman, D., \& Treisman, A. (1984). Changing views of attention and automaticity. In R. Parasuraman \& D. R. Davies (Eds.), Varieties of attention (pp. 29-61). New York: Academic Press.

Kahneman, D., \& Treisman, A., \& Burkell, J. (1983). The cost of visual filtering. Journal of Experimental Psychology, 9, 510-522.

KanTOWITZ, B. H. (1985). Channels and stages in human information processing: A limited analysis of theory and methodology. Journal of Mathematical Psychology, 29, 135-174.

Keren, G., O'Hara, W. P., \& Skelton, J. M. (1977). Levels of noise processing and attentional control. Journal of Experimental Psychology, 3, 653-664.

Kinchla, R. A. (1974). Detecting target elements in multielement arrays: A confusability model. Perception \& Psychophysics, 15, 149-158.

LABERGE, D. (1973). Attention and the measurement of perceptual learning. Memory \& Cognition, 1, 268-276.

LABERgE, D. (1975). Acquisition of automatic processing in perceptual and associative learning. In P. M. A. Rabbit \& S. Dornic (Eds.), Attention and performance $V$ (pp. 50-64). New York: Academic Press.

Lavie, N. (1995). Perceptual load as a necessary condition for selective attention. Journal of Experimental Psychology: Human Perception \& Performance, 21, 451-468.

Lavie, N., \& Tsal, Y. (1994). Perceptual load as a major determinant of the locus of selection in visual attention. Perception \& Psychophysics, 56, 183-197.

LogAN, G. (1988). Toward an instance theory of automatization. Psychological Review, 95, 492-527.

Miller, J. (1987). Priming is not necessary for selective-attention failures: Semantic effects of unattended, unprimed letters. Perception \& Psychophysics, 41, 419-434.

Miller, J. (1991). The flanker compatibility effect as a function of visual angle, attentional focus, visual transients, and perceptual load: A search for boundary conditions. Perception \& Psychophysics, 49, 270-288.

NAVON, D. (1989). The locus of attentional selection: Is it early, late, or neither? European Journal of Cognitive Psychology, 1, 47-68.

PASHLER, H. (1984). Evidence against late selection: Stimulus quality effects in previewed displays. Journal of Experimental Psychology: Human Perception \& Performance, 10, 429-448.

Rees, G., Frith, C. D., \& Lavie, N. (1997). Modulating irrelevant motion perception by varying attentional load in an unrelated task. Science, 278, 1616-1619.

Rees, G., Russell, C., Frith, C. D., \& Driver, J. (1999). Inattentional blindness versus inattentional amnesia for fixated but ignored words. Science, 286, 2504-2507.

Rohrbaugh, J. W., \& Eriksen, C. W. (1975). Reaction time measurement of temporal integration and organization of form. Perception \& Psychophysics, 17, 53-58.

SchmidT, P. A., \& DARK, V. J. (1999). Flanker recall and the flanker validity effect may reflect different attentional processes. Perception \& Psychophysics, 61, 424-437.
Shiffrin, R. M. (1988). Attention. In R. C. Atkinson, R. J. Herrnstein, G. Lindzey, \& R. D. Luce (Eds.), Stevens' Handbook of experimental psychology: Vol. 2. Learning and cognition (pp. 739-811). New York: Wiley.

Shiffrin, R. M., Diller, D., \& Cohen, A. (1995). Processing visual information in an unattended location. In A. F. Kramer, M. G. H. Coles, \& G. D. Logan (Eds.), Converging operations in the study of visual selective attention (pp. 225-246). Washington DC: American Psychological Association.

ShIFFrin, R. M., \& GARDNER, G. T. (1972). Visual processing capacity and attentional control. Journal of Experimental Psychology, 93, $72-82$.

Shiffrin, R. M., \& SchneIder, W. (1977). Controlled and automatic human information processing: II. Perceptual learning, automatic attending and a general learning theory. Psychological Review, 84, 127-190.

SPERLING, G. (1960). The information available in brief visual presentations. Psychological Monographs, 74, 498.

Stelmach, L. B., \& Herdman, C. M. (1991). Directed attention and perception of temporal order. Journal of Experimental Psychology: Human Perception \& Performance, 17, 539-550.

Stelmach, L. B., Herdman, C. M., \& McNeil, R. K. (1994). Attentional modulation of visual processes in motion perception. Journal of Experimental Psychology: Human Perception \& Performance, 20, 108-121.

TIPPER, S. P. (1985). The negative priming effect: Inhibitory effects of ignored primes. Quarterly Journal of Experimental Psychology, 37A, 571-590.

Tipper, S. P., \& CRAnSTON, M. (1985). Selective attention and priming: Inhibitory and facilitatory effects of ignored primes. Quarterly Journal of Experimental Psychology, 37A, 591-611.

Treisman, A., \& Gelade, G. (1980). A feature-integration theory of attention. Cognitive Psychology, 12, 97-136.

YANTIS, S., \& Johnston, J. C. (1990). On the locus of visual selection: Evidence from focused attention tasks. Journal of Experimental Psychology: Human Perception \& Performance, 16, 135-149.

\section{NOTES}

1. Duration of the events within trials were based on program timing specifications and the CRT refresh rate. All timing was confirmed by an oscilloscope and software that provided a listing of the times at which commands were executed and times at which the raster scan was at the top of the screen.

2. We utilized more salient cues than Eriksen et al. (1990; dashes around the letter as opposed to a line underscoring the letter), which may have led to some letter masking or a general distraction effect, especially when cues were closer in proximity (Fournier, 1994; Kahneman, Treisman, \& Burkell, 1983).

3 . The small variability in durations of events within a trial between the two experiments is based on the variability in CRT refresh rates between the two studies.

(Manuscript received January 25, 2000; revision accepted for publication January 12, 2001.) 